\title{
Modélisation numérique de l'hydrodynamique avec interaction houle-courant : Effet sur le taux de cisaillement au fond
}

\author{
Laila MOUAKKIR ${ }^{1}$, Hassan SMAOUI ${ }^{2}$, Soumia MORDANE ${ }^{1}$, Mohammed \\ CHAGDALI ${ }^{1}$
}

'Laboratoire de Calcul Scientifique en Mécanique, Faculté des Sciences Ben M'Sik, Université Hassan II- Mohammedia, Casablanca.

mouakkir 1aila@yahoo.fr

${ }^{2}$ Département de Mathématiques et Informatique, Ecole Nationale Supérieure des Arts et des Métiers- Méknès.

\section{Résumé}

Dans ce travail on s'intéresse à une modélisation numérique qui permet de décrire la prise en compte de la houle dans un écoulement généré par les courants de marée. Notre contribution à cette étude a été réalisée à partir de deux modèles : le modèle MECCA, et le modèle SWAN. L'interaction houle-courant a été incluse. Les résultats entrepris dans ce travail montrent l'effet de la superposition de la houle et du courant sur le cisaillement au fond par rapport au courant seul. Autrement dit, près du fond, l'effet de la houle augmente la turbulence, de ce fait, le cisaillement et le frottement augmentent en entraînant une réduction de la vitesse près du fond.

Mots-clés :

MECCA - SWAN - Interaction houle courant

\section{Introduction :}

La zone côtière (plaine côtière et plateau continental) ne représente que $8 \% \mathrm{du}$ système planétaire, mais intègre les écosystèmes les plus productifs et les plus diversifiés. Par ailleurs, l'activité humaine s'exerce en particulier dans le secteur côtier. La connaissance de cette frange s'avère donc indispensable. La complexité des processus intervenant dans la circulation océanique côtière nécessite l'utilisation de la modélisation numérique.

Dans le but d'examiner l'impact de l'interaction houle-courant sur l'hydrodynamique, nous proposons dans cette étude, une modélisation numérique 
permettant de d'écrire la prise en compte de la houle dans un écoulement généré par les courants de marée. Le code numérique MECCA sera un outil dans cette modélisation. C'est un code 3D simulant les écoulements côtiers et quelques processus hydro-sédimentaires ( transport sédimentaire, qualité des eaux...). Ce code ne tient pas compte de l'influence de la houle. D'ailleurs, la houle est un des principaux facteurs responsables de la mise en suspension du sédiment. Il est donc irréaliste de négliger ce phénomène pour l'étude du transport en zone côtière. Pour cela, nous optons pour une paramétrisation de l'interaction houle-courant. Les paramètres de la houle utilisés dans cette approche sont calculés à partir du code SWAN. A partir du courant simulé par le modèle MECCA, et d'un champ de houle prédit par SWAN, nous explicitons le frottement lié à l'interaction de ces phénomènes. Ce frottement est responsable de l'augmentation de l'énergie cinétique turbulente au fond et par conséquent de la remise en suspension des sédiments. Dans ce travail, nous allons nous intéresser à l'influence de l'interaction houle-courant sur l'hydrodynamique, plus particulièrement sur l'effet de la houle sur le taux de cisaillement au fond

\section{$2 \quad$ Présentation des modèles numériques}

\subsection{Modèle de l'hydrodynamique (MECCA)}

Le noyau de ce modèle (version 2D) a été développé initialement au sein de la N.O.A.A (National Oceanic and Atmospheric Administration ) par (HESS, 1986). Le modèle permet de simuler l'évolution de la marée, de la température, de la salinité et des sédiments en suspension. Sa résolution numérique utilise les approximations de Boussinesq, hydrostatique et du plan Beta. Il permet également de simuler les écoulements générés par le vent, les gradients de la densité et les gradients de le pression atmosphérique. Les équations du modèle $\mathrm{s}$ 'expriment dans un repère orthonormé $(\mathrm{x}, \mathrm{y}, \mathrm{z})$ par :

$$
\begin{aligned}
& \frac{\partial u}{\partial t}+\vec{V} \cdot \nabla u-f v=-\frac{1}{\rho_{o}} \frac{\partial p}{\partial x}+\frac{\partial}{\partial z}\left(A_{v} \frac{\partial u}{\partial z}\right)+F_{u} \\
& \frac{\partial v}{\partial t}+\vec{V} \cdot \nabla v-f u=-\frac{1}{\rho_{o}} \frac{\partial p}{\partial y}+\frac{\partial}{\partial z}\left(A_{v} \frac{\partial v}{\partial z}\right)+F_{v}
\end{aligned}
$$

Avec

$$
\begin{aligned}
& F_{u}=\frac{\partial}{\partial x}\left(2 A_{h} \frac{\partial u}{\partial x}\right)+\frac{\partial}{\partial y}\left[A_{h}\left(\frac{\partial u}{\partial y}+\frac{\partial v}{\partial x}\right)\right] \\
& F_{v}=\frac{\partial}{\partial x}\left(2 A_{h} \frac{\partial v}{\partial y}\right)+\frac{\partial}{\partial x}\left[A_{h}\left(\frac{\partial u}{\partial y}+\frac{\partial v}{\partial x}\right)\right]
\end{aligned}
$$


Où $u, v, w$ sont les composantes du vecteur vitesse $\vec{V}, p$ est la pression , $f$ est le paramètre de Coriolis, $\rho_{\dot{a}}$ est une densité de référence $A_{v}, A_{h}$ est la viscosité turbulente verticale et horizontale. L'approximation hydrostatique permet d'écrire :

$$
\rho g=-\frac{\partial p}{\partial z}
$$

L'hypothèse d'incompressibilité permet de réduire l'équation de la continuité à :

$$
\frac{\partial u}{\partial x}+\frac{\partial v}{\partial y}+\frac{\partial w}{\partial z}=0
$$

Les coefficients de mélange $A_{v}, A_{h}$ sont calculés par un modèle de la turbulence à une équation donné par le système suivant :

$$
\frac{\partial K}{\partial t}+\vec{V} . \nabla K=A_{v}\left[\left(\frac{\partial u}{\partial z}\right)^{2}+\left(\frac{\partial v}{\partial z}\right)^{2}\right]+F_{K}+\frac{\partial}{\partial z}\left(A_{K} \frac{\partial K}{\partial z}\right)+\frac{g}{\rho_{0}} A_{h} \frac{\partial \rho}{\partial z}-c_{\mu} \frac{K^{1.5}}{L}
$$

$K$ est l'énergie cinétique turbulente (ECT), $L$ est la longueur de mélange, $c_{\mu}$ est une constante empirique, $F_{k}$ est le flux horizontal de l'ECT dont l'expression est analogue à celle donnée par l'équation (3). L'échelle de longueur L sera prescrite de la façon suivante ( BLAKHADAR,1962) :

$L=\frac{\kappa z L_{0}}{L_{0}+\kappa z} \quad L_{0}=\gamma \frac{\int_{z 0}^{\infty} z \sqrt{K} \cdot d z}{\int_{z 0}^{\infty} \sqrt{K} d z}$

Avec $\gamma$ est une constante empirique variant de 0.05 et $0.3, z_{0}$ est la longueur de la rugosité du fond, $\kappa$ est la constante de Karman $(\kappa=0.4)$. Pour fermer le système d'équations, les coefficients $A_{v}, A_{h}$ sont donnés par :

$$
A_{v}=S_{a v} L \sqrt{K} \quad A_{h}=S_{a h} L \sqrt{K} \quad A_{v}=S_{a k} L \sqrt{K}
$$

$S_{a v}, S_{a h}, S_{a k}$ sont des fonctions de stabilités (BLUMBERG \& MELLOR, 1987) Pour une discussion détaillée du modèle MECCA, le lecteur consultera les références (SMAOUI, 1996 et BERTHET 1996).

\subsection{Modèle spectrale des vagues}

Le modèle SWAN (Simulating Waves Nearshore) (BOOJ, et al 1999) (version4041) est un modèle numérique qui permet d'obtenir des estimations des caractéristiques des vagues dans les zones côtières, pour des conditions de vent, courant et bathymétrie connus. Le modèle est basé sur l'équation de conservation de la densité d'action des vagues. SWAN est exécuté en mode troisième génération, stationnaire et bidimensionnel. Les paramètres de sortie SWAN utilisés dans ce travail sont la hauteur significative et la période moyenne. 


\subsection{Modèle d'interaction houle- courant}

Comme le montre les simulations numériques réalisées par (DAVIES et LAWRENCE, 1994,1995), l'interaction houle-courant joue un rôle important dans la circulation côtière, autrement dit, en présence de la houle, l'augmentation de la turbulence influence à son tour l'écoulement au voisinage de la couche limite. Cette modification sera contenue dans le coefficient de frottement seul et par conséquent on aboutit à une modification des contraintes de cisaillements au fond. Dans ce document, la procédure de l'interaction houle-courant proposée par (DAVIES \& LAWRENCE, 1994,1995 ; SIGNELL et al 1990) a été adoptée.

\section{Application des modèles à Casablanca-Mohammedia (Maroc)}

Le secteur étudié est situé à l'ouest du Maroc sur le littoral atlantique entre les longitudes $7^{\circ} 20^{\prime}$ et $7^{\circ}, 51^{\prime}$ ce qui correspond à une longueur $31^{\prime}$ ou $57350 \mathrm{~m}$, et les latitudes $33^{\circ} .30^{\prime}$ et $33^{\circ} .47^{\prime}$ c'est à dire une largeur de $17^{\prime}$ ou $31450 \mathrm{~m}$. Les données de la bathymétrie sont obtenues à partir d'une carte bathymétrique fournie par la direction des ports de Casablanca-Mohammedia (figure 1). Pour obtenir une bathymétrie sur une grille régulière de pas spatial $1 \mathrm{~km}$; nous avons utilisé un logiciel de digitalisation "Digit", puis un logiciel d'interpolation "Krigeage".

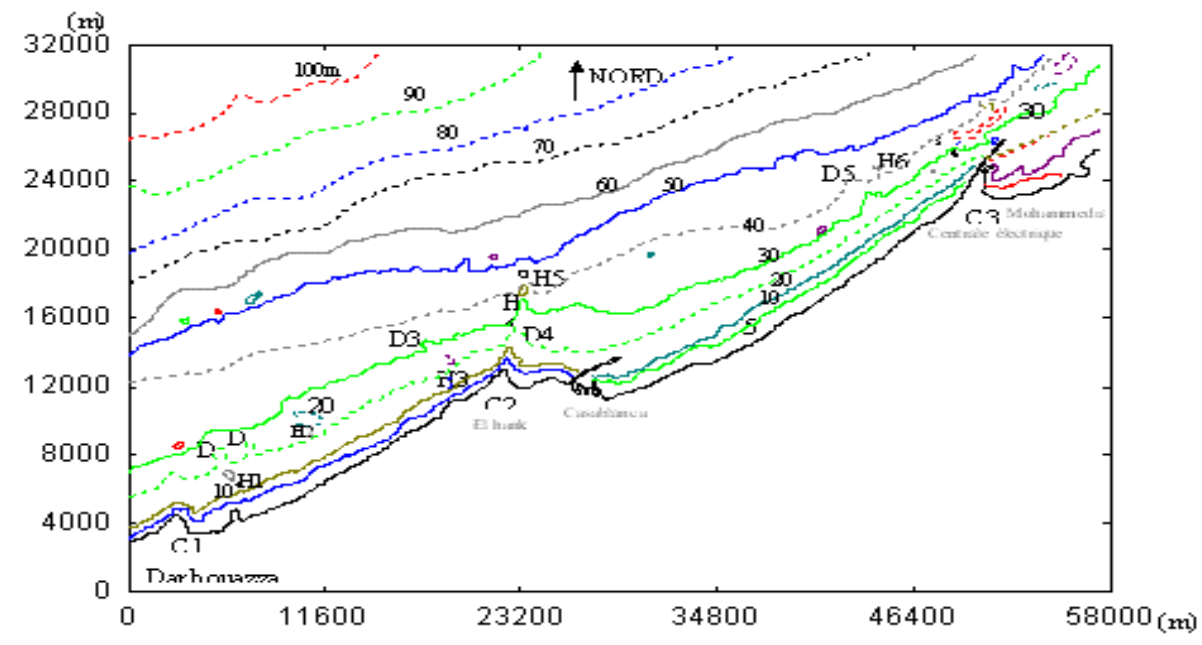

Figure 1: Carte de la bathymétrie au niveau de la zone côtière s'étendant de Dar Bouaaza à Mohammedia

* Les simulations numériques de la houle sur le littoral Casablanca-Mohammedia ont été réalisées en s'appuyant sur le cas d'hiver avec une houle en provenance du large de direction NW ayant une hauteur de $4 m$ et une période de 14 secondes. 
* La marée dominante dans ce secteur est de type semi-durne M2 de période $T=$ $12.4 \mathrm{~h}$, d'amplitude $2 \mathrm{~m}$, telle qu'affiché sur les frontières ouvertes du domaine. Nous avons utilisé un logiciel de la marée mondiale, à partir duquel nous avons extrait, en une série de 5 points répartis sur les frontières ouvertes, la hauteur d'eau sous forme de série temporelle couvrant une période de marée (12.4h).

\section{$4 \quad$ Résultats \& discussion}

\subsection{Application du code SWAN}

Les simulations réalisées par le code SWAN montrent que la hauteur significative de la houle diminue lors de son déplacement depuis le large à la côte. L'effet du fond commence à se faire sentir à l'approche du rivage et au voisinage des dorsales sous marine et des hauts fonds. La figure 2 montre un accroissement de la hauteur de la houle (qui passe de moins $3.8 \mathrm{~m}$ à $4.5 \mathrm{~m}$ ) au voisinage de Dar bouazza et précisément aux alentours des dorsales D1 et D2 ainsi que des hauts fonds $\mathrm{H} 1$ et $\mathrm{H} 2$. La même remarque est valable au cap El Hank surtout dans sa façade Ouest ce qui correspond à l'effet de la dorsale D3 et du haut fond H3.

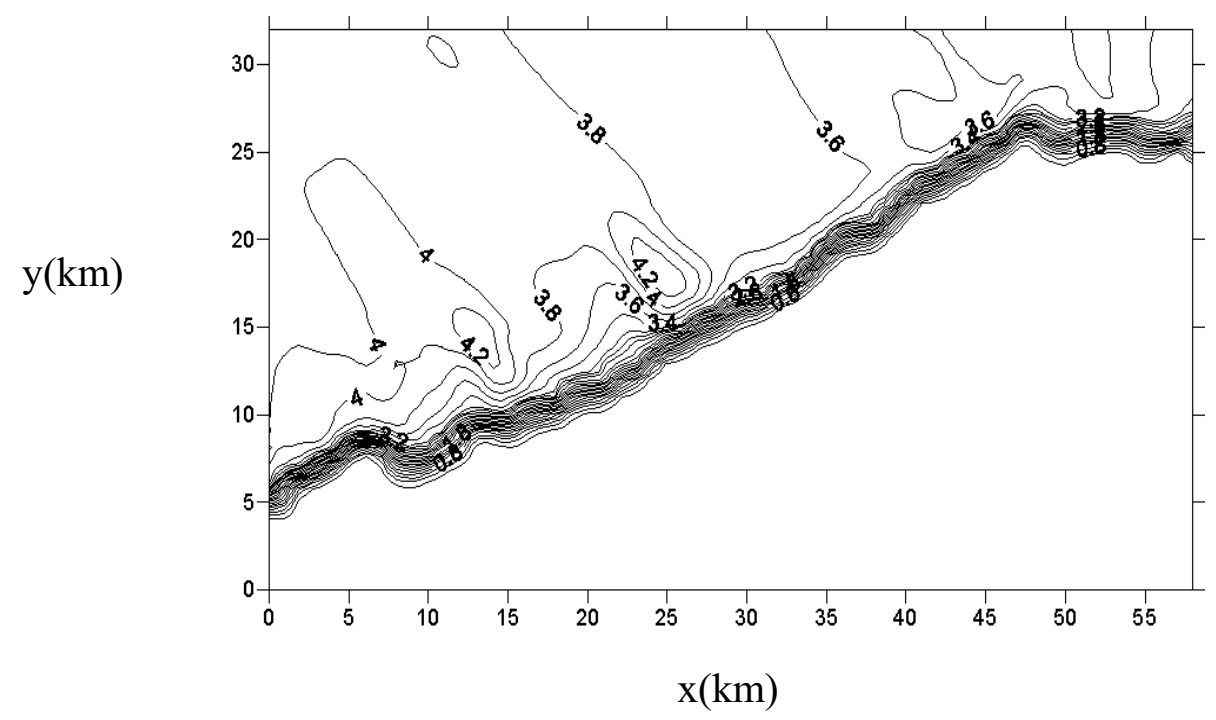

Figure 2 Distribution spatiale de la hauteur significative de la houle, simulée par le modèle SWAN

\subsection{Application MECCA+SWAN}

Les figures $(3,4)$ présentent respectivement les profils verticaux de l'énergie cinétique turbulente (ECT) et de la composante $u$ de la vitesse $U(u, v, w)$ toutes les heures de simulation pendant un cycle de marée au point situé au cap El 
Hank. Nous remarquons près du fond, une augmentation de l'ECT et une réduction de la vitesse. La figure 5 présente la variation temporelle correspondant respectivement au taux de cisaillement et à l'ECT, ces courbes montrent une croissance remarquable dans le cas de superposition houle/courant par rapport au courant seul. Autrement dit, l'effet de la houle au fond augmente la turbulence, de ce fait, le cisaillement et le frottement augmentent. Ceci et en parfait accord avec la bibliographie à ce sujet (DAVIES,A.M and LAWRENCE, J 1994, 1995) 

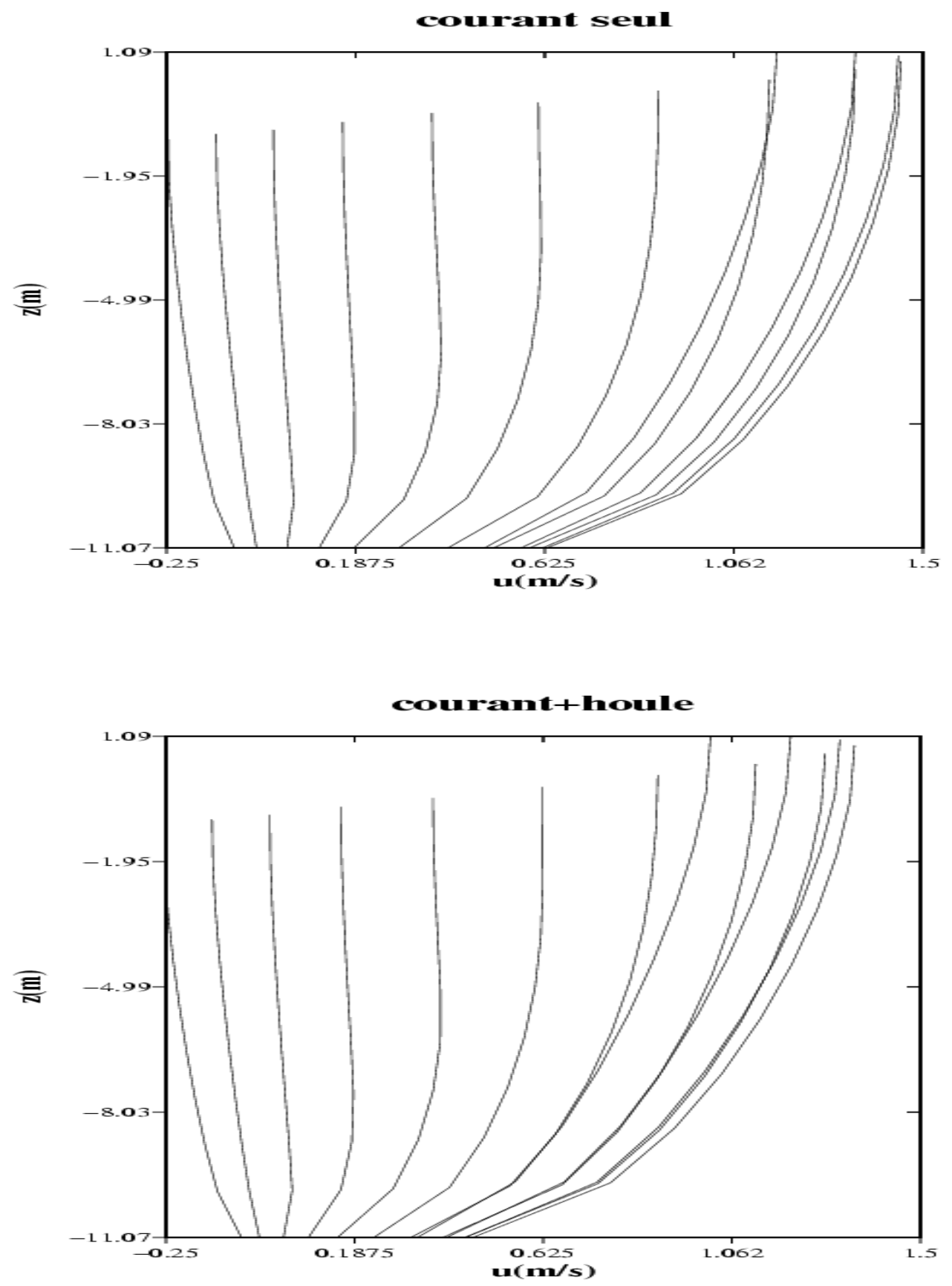

Figure3 Profils verticaux de la composante $u$ de la vitesse pendant un cycle de marée 

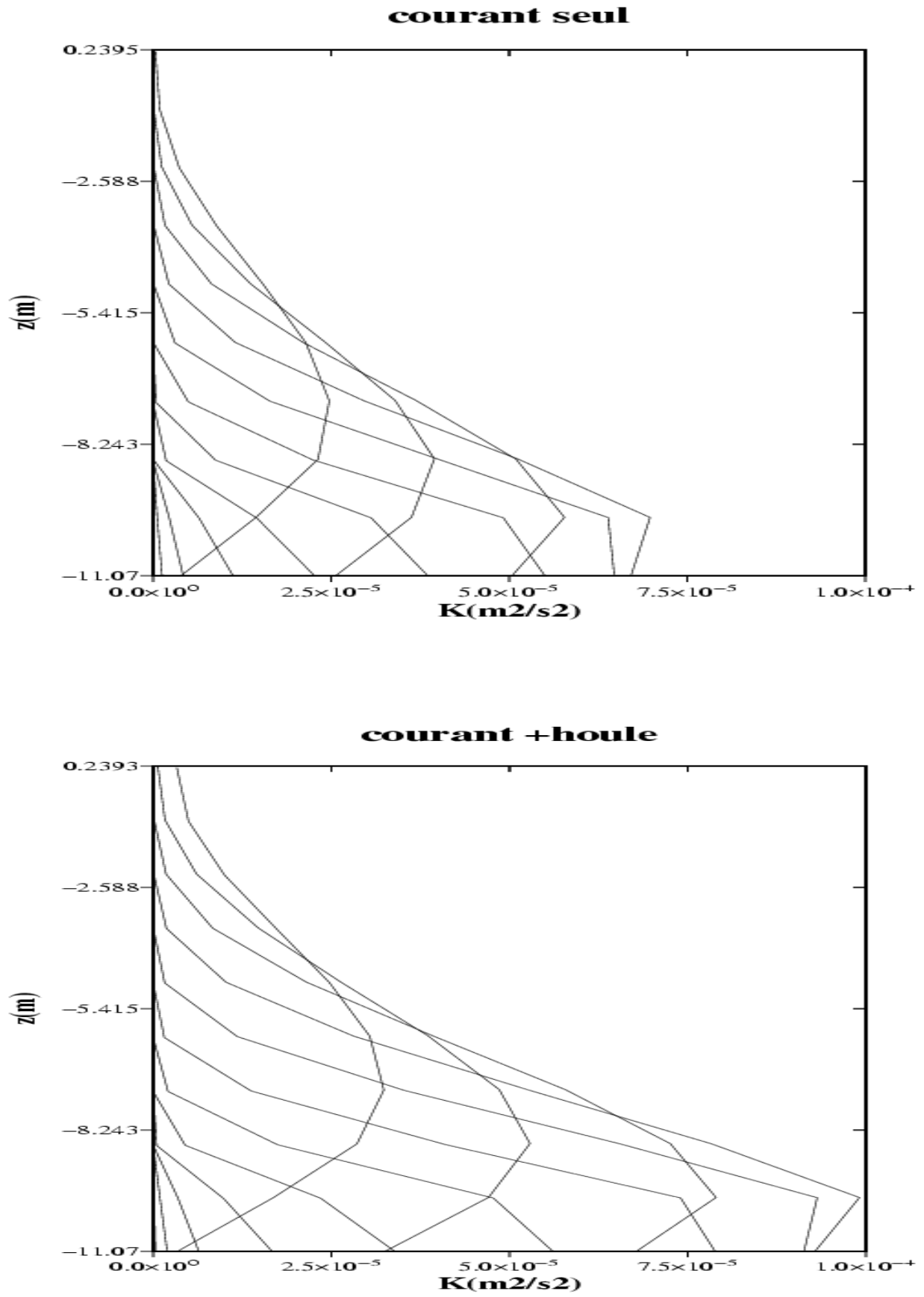

Figure4 Profils verticaux de l'énergie cinétique turbulente ECT pendant un cycle de marée 

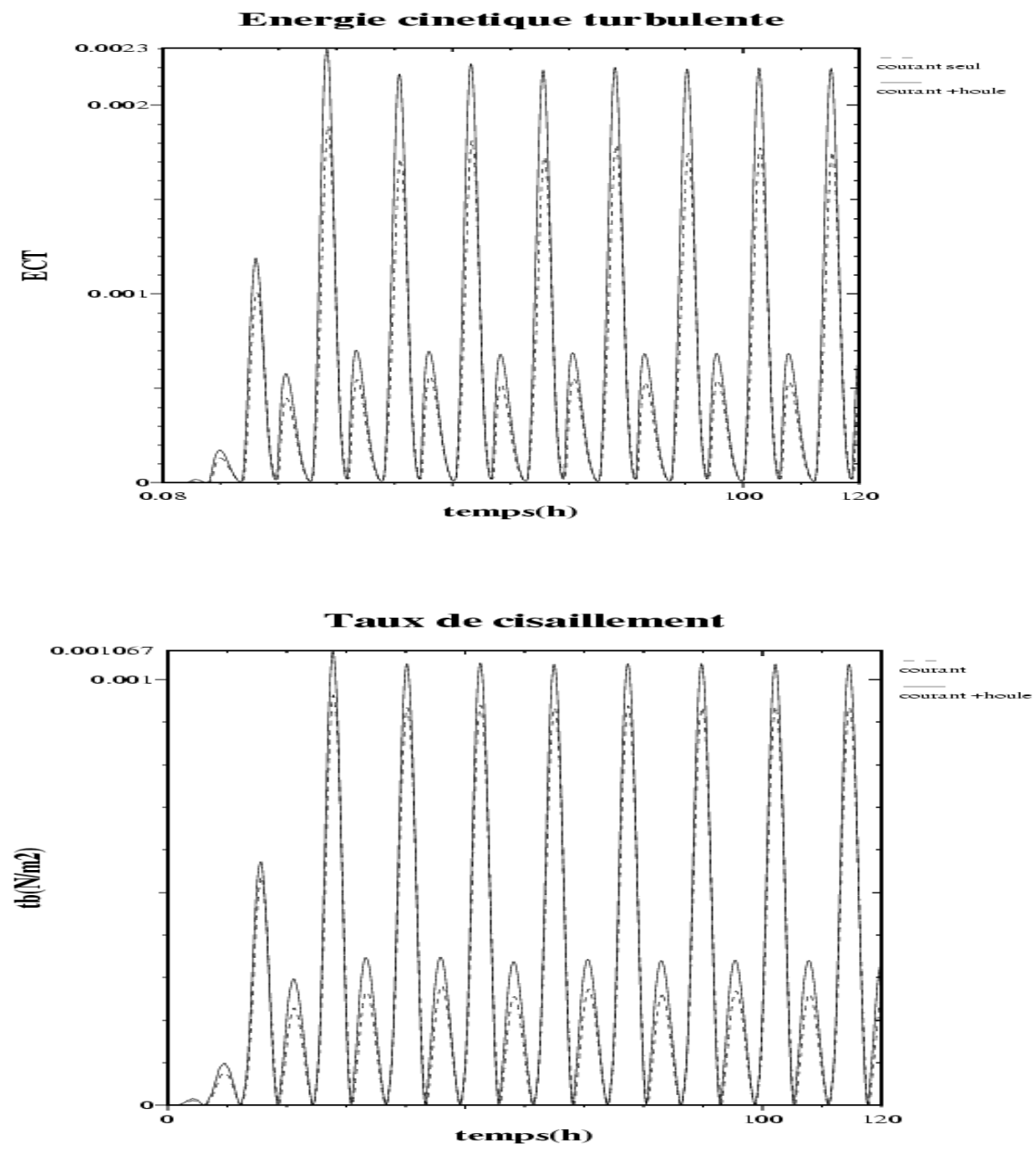

Figure 5 Variation temporelle de l'ECT et du taux de cisaillement au fond

\section{Conclusion}

Au cours de cette étude, nous avons effectué une modélisation tridimensionnelle examinant l'influence de l'interaction houle-courant sur l'hydrodynamique. La mise au point de cette modélisation est basée sur deux codes numériques : MECCA et SWAN. La paramétrisation de l'interaction houle-courant est basée sur l'approche de (DAVIES,A.M \& LAWRENCE, J 1994, 1995). Les résultats de cette modélisation montrent l'influence de la combinaison houle/courant sur le cisaillement au fond par rapport au courant seul. Autrement dit, près du fond, l'effet de la houle augmente la turbulence, par suite, le cisaillement et le frottement augmentent en entraînant une réduction de la vitesse près du fond. 
Nous nous proposons de poursuivre cette étude de manière à introduire le transport sédimentaire et par suite examiner l'effet de la houle sur la suspension des sédiments.

\section{$6 \quad$ Références bibliographiques}

1 BOOJ, N., RIS, R., HOLTHUIJSEN, L., 1999. A third-generation wave model for coastal regions, part : Model description abnd validation. Journal of Geophysical Research 104 (C4), 76497666.

2 BERTHET.C, 1996 : Ecoulements et transports littoraux tridimensionnels : application numériques. thèse de doctorat de l'Université Joseph FourierGrenoble 1

3 BLAKHADAR, A.K, 1962: The vertical distribution of wind and turbulent exchange in a neutral atmosphere. J.Geophys.Res,67,pp 3095-3120

4 BLUMBERG, A.F and G.L.MELLOR ,1987: A description of a threedimensional coastal ocean circulation model, in "three-dimensional coastal ocean models". Coastal and Estuarine Sciences 4. American Geophysical Union, Washington DC, pp 1-16

5 DAVIES,A.M and LAWRENCE, J 1994: Examining the influence of Wind Wave Turbulence on Tidal Currents, Using a Three-Dimensional Hydrodynamique Model Including Wave-Current Interaction. Journal of Physical Oceanography, Volume 24, pp 2441-2460.

6 DAVIES, A.M and LAWRENCE, J 1995 Modelling the effect of wave current interaction on the Three- Dimensional Hydrodynamique wind driven circulation of the eastern Irish Sea. Journal of Physical Oceanography,25 29-45.

7 HESS, K, W. 1986 : Numérical model of circulation in Chesapeake bay and the continental shelf, N.O.A.A Technical Memorandum, AISC6, U.S Dpt of commerce, pp 47

8 SMAOUI, H 1996: Modélisation numérique tridimensionnelle de l'hydrodynamique et des transports sédimentaires en Manche Orientale et dans le sud de la Mer du Nord, thèse de doctorat de l'Université des Sciences et Technologies de Lille, France

9 SIGNELL, R, P., R. C. BEARDSLEY, H.C. GRABER, and A. CAPOTONDI, 1990: Effect of wave-current interaction on wind-driven circulation in narrow, shallow embayment. J. Geophys. Res., 95, 9671-9678 